\title{
Simulación de la eficacia de ignífugos inorgánicos en la extinción de incendios con CFAST. Caso de un laboratorio químico.
}

Simulation of the effectiveness of inorganic flame retardants in extinguishing fires with CFAST. Case of a chemical laboratory.

\author{
Manolo Alexander Córdova Suárez. ${ }^{1}$, Danielita Fernanda Borja Mayorga. ${ }^{2}$, Edison \\ Patricio Villacres Cevallos. ${ }^{3} \&$ Montes Vega Klever Saul. ${ }^{4}$
}

Abstract.

DOI: https://doi.org/10.33262/concienciadigital.v4i1.2.1587

Introduction. The use of inorganic flame retardants in sprinkler-type fire systems is an alternative that can help in extinguishing uncontrolled fires and protecting high-risk chemical laboratories. Objetives. Extinguishing conditions were established according to NFPA 13 and UNE 12845 standards and the efficacy was calculated by comparing the two conditions: use of water and use of inorganic fire retardant, using the Consolidated Model of Fire and Smoke Transport (CFAST) and Smokview 7 software. Methodology. It was determined: the level of fire risk according to the UNE 12845 standard, then the operating conditions under critical operating conditions and maximum work load according to the NFPA 13 and UNE 12845 standards and finally the effectiveness of smoke evacuation and reduction was tested of the temperature of the room by testing the inorganic fire retardant solution with known values of decrease in the heat release curve (HRR) with the CFAST and Smkeview 7 program. Results. It was determined: extragroup 2 risk fire risk level, the maximum surface area per sprinkler is $12 \mathrm{~m} 2$, the minimum design density is $5 \mathrm{~mm} / \mathrm{min}$ for each sprinkler, the smoke saturation improvement time of the site considering the use of water and dilution of inorganic flame retardants based

\footnotetext{
${ }^{1}$ G+ Energy-Risks \& Engineering Group, Department of Food Science and Engineering, Technical University of Ambato, Ambato-Ecuador, ma.cordova@uta.edu.ec, orcid.org/0000-0001-6786-7926

2 Faculty of Chemical Engineering, Higher Polytechnic School of Chimborazo, Riobamba-Ecuador, dborja@ spoch.edu.ec, orcid.org/0000-0002-8438-064X

3 Faculty of Engineering, National University of Chimborazo, Riobamba-Ecuador, pvillacres@unach.edu.ec, orcid.org/0000-0001-9518-1278

${ }^{4}$ University of Guadalajara, Guadalajara-México, klever.montes9254@alumnos.udg.mx, orcid.org/00000002-3573-4018
} 
on Magnesium Hydroxide $\mathrm{Mg}(\mathrm{OH}) 2$ at 9\% (Pozo Álvarez, 2020) as an extinguishing medium is $37 \%$ and a $48.57 \%$ improvement in cooling time is observed, with a pressure of $101,500 \mathrm{~Pa}$, an average temperature of $20^{\circ} \mathrm{C}$ in the city of Ambato, an oxygen percentage of 15\% according to the NFPA 13 and UNE 12845 standards. Conclusion. The application of inorganic flame retardants Magnesium Hydroxide as a fire extinguishing method results in a $37 \%$ improvement in the increase in the time in the removal of pollutants and a $48.57 \%$ improvement in the cooling time assumes an improvement in system efficiency according to CFAST.

Keywords: CFAST, smoke, sprinklers, SMOKEVIEW, fires.

\section{Resumen.}

Introducción. El uso de ignífugos inorgánicos en sistemas contraincendios tipo rociadores es una alternativa que puede ayudar en la extinción de fuegos no controlados y la protección en laboratorios químicos con alto riesgo. Objetivo. Se estableció las condiciones de extinción según normas NFPA 13 y UNE 12845 y se calculó la eficacia por comparación de las dos condiciones: uso de agua y uso de ignífugo inorgánico, utilizando el software Consolidated Model of Fire and Smoke Transport (CFAST) y Smokview 7. Metodología. Se determinó: el nivel de riesgo de incendio según la norma UNE 12845, luego las condiciones de operación en condiciones críticas de funcionamiento y en carga de trabajo máximo según norma NFPA 13 y UNE 12845 y finalmente se probó la eficacia del desalojo de humo y disminución de la temperatura del recinto probando la solución ignífuga inorgánica con valores conocidos de disminución en la curva de liberación de calor (HRR) con el programa CFAST y Smkeview 7. Resultados. Se determinó: nivel de riesgo de incendio riesgo extra-grupo 2, la superficie máxima por rociador es de $12 \mathrm{~m}^{2}$, la densidad de diseño mínimo es $5 \mathrm{~mm} / \mathrm{min}$ por cada sprinkler, el tiempo de mejora de saturación de humo del emplazamiento considerando el uso de agua y de dilución de ignífugos inorgánicos a base de Hidróxido de Magnesio $\mathrm{Mg}(\mathrm{OH})_{2}$ al 9\% (Pozo Álvarez, 2020) como medio de extinción es 37\% y se observa un $48,57 \%$ de mejora en el tiempo de enfriamiento, con una presión de $101500 \mathrm{~Pa}$, una temperatura promedio de $20{ }^{\circ} \mathrm{C}$ en la ciudad de Ambato, un porcentaje de oxígeno del 15\% según la norma NFPA 13 y UNE 12845. Conclusión. La aplicación de ignífugos inorgánicos Hidróxido de Magnesio como método de extinción de incendios resulta en un $37 \%$ de mejora en el incremento del tiempo en el desalojo de los contaminantes y un $48,57 \%$ de mejora en el tiempo de enfriamiento, por los resultados se asume una mejora en la eficiencia del sistema según CFAST.

Palabras claves: CFAST, humo, sprinklers, SMOKEVIEW, incendios.

\section{Introducción.}

The Los incendios son desastres naturales y en varios casos son inducidos por el hombre. Durante los últimos años han aumentado su frecuencia, causando daños irremediables en 
vidas humanas y en pérdidas materiales. A la par se ha desarrollado la ingeniería de incendios cuyo objetivo es brindar maneras de extinción que atenúen estos eventos no deseados que provocan pérdidas irreparables y daños cuantiosos.(Purasinghe et al., 2021)

La ingeniería de incendios fundamenta su accionar en el uso de métodos de extinción de acuerdo al tipo de incendio y las condiciones que lo permiten, pero estos ensayos y pruebas que predicen el comportamiento del incendio y ayudan a brindar mejores alternativas preventivas son muy costosas y se demoran en su ejecución por lo que se recurre al uso de software y herramientas informáticas que estimen las consecuencias y den una visión del incendio si este ocurriera (CITA). La modelación de incendios fundamenta su accionar en cálculos matemáticos basados en los modelos físicos del evento llevados a una discretización de los datos para al final validar los resultados con estudios reales (Cohen et al., 2019).

Estos modelos de incendios simulan las condiciones de trabajo y calculan temperaturas, concentraciones de contaminantes productos de la combustión e incluso daño estructural o mecánico de los materiales constructivos pero fundamentalmente existen ciertas aplicaciones como Consolidated Model os Fire Growith and Smoke Transport (Vadlamuri y Lilley, 2009) que incorpora sistemas de prestaciones para el control de incendios como splinkers, alarmas y sensores de temperatura que ayudan significativamente en una toma decisiones al momento de evaluar un evento no deseado y saber el comportamiento de las capas de temperaturas y el movimiento del humo en cada compartimiento de una manera visual (Vadlamuri y Lilley, 2010).

Previo a la simulación del incendio el cálculo de las prestaciones representa una parte crucial en la elección del tipo de sistema de extinción y utiliza sus recomendaciones considerando el tipo de nivel de riesgo de incendios que presenta la norma UNE 12845 en sus apartados sobre, diseño instalación y mantenimiento de los sistemas de rociadores automáticos (Runefors et al., 2018). Las condiciones de trabajo que requiere el CFAST para la simulación toma en cuenta entre algunos datos: presión atmosférica, temperatura interna y externa, \% de oxígeno, datos fisicoquímicos del tipo de paredes, techos, puertas, ventanas, pisos, conexiones, aperturas de puertas y ventanas preestablecidas y principalmente el sistema de extinción (Mikhailovsky, 2020).

El CFAST brinda como resultados finales una tabla de cantidades en intervalos de tiempo seleccionados, además los tiempos de activación de los sistemas contra incendios colocados en cada recinto. Para establecer de manera visual el programa Smokview 7 establece el comportamiento de temperaturas, materiales formados en el incendio como productos de combustión y figuras crecientes y decrecientes de los fuegos según se coloquen en los emplazamientos en función del tiempo de la simulación. (Huang et al., 2012)

Este trabajo conlleva a pruebas de comportamiento de las dos capas de temperatura en el incendio y el desalojo del humo en los habitáculos del recinto estudiado considerando una disminución de 8 (Canosa y Giudice, 2016) en la curva de crecimiento de calor (HRR) de los materiales que se utilizan como combustibles o mobiliario que ocupa el personal 
del Laboratorio de Control y Análisis de Alimentos de la Universidad Técnica de Ambato.

\section{Metodología}

Para el diseño de un sistema de protección se calculó las siguientes condiciones:

\section{Determinación del área total del emplazamiento}

Se determinó dimensiones de largo, ancho y altura, de todas los recintos de LACONAL, posteriormente se calculó el área total o área de operación como se muestra en la Tabla 3 considerando las áreas limítrofes $A_{\mathrm{L}} \mathrm{y}$ áreas de apertura $\mathrm{A}_{\mathrm{W}}$ de cada dependencia con las fórmula de la ecuación 1, 2 y 3. (Zurita Castro, 2020)

Ecuación 1: $A_{T}=A_{L}-A_{w}$

Ecuación 2: $A_{L}=2 * D * W+2 h(D+W)$

\section{Donde:}

$A_{L}=$ Area limitrofe $\left(\mathrm{m}^{2}\right)$

$D=\operatorname{Largo}(m)$

$W=\operatorname{ancho}(m)$

$h=\operatorname{Altura}(m)$

Ecuación 3: $A_{w}=H * B$

Donde:

$A_{w}=$ Área de aperturas $\left(\mathrm{m}^{2}\right)$

$H=$ Largo de la apertura $(m)$

$B=$ Ancho de la apertura $(m)$

Categorización del nivel de riesgo en el sector del incendio.

Para establecer las condiciones de extinción por agua mediante la utilización de rociadores se definió la clasificación del riesgo del laboratorio según la UNE 12845 y comprobando diferencias con la Norma NFPA 13. Se identificó a LACONAL riesgo leve, riesgo ordinario grupo 1, riesgo ordinario grupo 2, riego extra-grupo 1, riesgo extra-grupo 2. (Grill, 2018)

\section{Determinación del caudal total de rociadores}

Con el riesgo identificado y el área de diseño se obtuvo el valor de la densidad de cobertura del rociador y posteriormente se aplicó la ecuación 4 para el cálculo de caudal de agua de los rociadores (Saavedra Castro y Rosales España, 2016):

Ecuación 4: $Q_{T R}=A d * \rho$

\section{Donde:}

$Q_{T R}=$ Caudal total de rociadores $(L / \mathrm{min})$

$A d=$ Área de operacion $\left(m^{2}\right)$ 
$\rho=\operatorname{Densidad}\left(L / \mathrm{min} / \mathrm{m}^{2}\right)$

\section{Cálculo del número de rociadores en el área de diseño}

Se tomó de referencia la figura 4. de la NFPA 13, considerando_ rociadores convencionales debido a y una presión de 75 psi, se calculó con la ecuación 5 el número de rociadores (Willis y Llaja, 2020) así:

$$
\text { Ecuación 5: Numero de Rociadores }=\frac{Q_{T R}}{Q_{R}}
$$

\section{Donde:}

$Q_{T R}=$ Caudal total de rociadores $(\mathrm{L} / \mathrm{min})$

$Q_{R}=$ Caudal de rociadores $(L / \mathrm{min})$

\section{Cálculo del caudal total del sistema}

El caudal total del sistema se determinó utilizando la ecuación 6. así:

$$
\text { Ecuación 6: } Q_{\text {total }}=\left(1.15 * Q_{T R}\right)+Q_{\text {mang }}
$$

\section{Donde:}

$Q_{T R}=$ Caudal total de rociadores $(\mathrm{L} / \mathrm{min})$

$Q_{\text {mang }}=$ Caudal de las mangueras $(L / \mathrm{min})$

\section{Determinación del volumen de agua para la fuente de abastecimiento}

Para determinar la capacidad de la fuente de abastecimiento (MANSILLA, 2014), se utilizó la ecuación 7. así:

$$
\text { Ecuación 7: } \mathrm{V}=\mathrm{Q}_{\text {total }} * t
$$

Donde:

$\mathrm{V}=$ Volumen $(\mathrm{L})$

$\mathrm{Q}_{\text {total }}=$ Caudal total del sistema $(L / \mathrm{min})$

$\mathrm{t}=$ Tiempo (min)

\section{Simulación con CFAST}

Para llevar a cabo la simulación en CFAST en la pestaña Simulation se detalló ciertos datos como: a) Tiempo de simulación en segundos, b) Intervalo de salida de texto, c) Intervalo de salida de la hoja de cálculo, d) Intervalo de salida de vista de humo, e) Temperatura interior y exterior, humedad y presión. Subsecuentemente, en la pestaña de Thermal Properties se colocó valores como densidad en $\mathrm{kg} / \mathrm{m} 3$, conductividad térmica en $\mathrm{kW} /\left(\mathrm{m}{ }^{\circ} \mathrm{C}\right)$, calor específico en $\mathrm{kJ} /\left(\mathrm{kg}{ }^{\circ} \mathrm{C}\right)$, espesor en $\mathrm{m}$ y emisividad (adimensional) de cada material de construcción tomados a partir de características térmicas de los materiales de construcción de la Norma Ecuatoriana de la construcción (Portilla et al., 
2006) para cada material agregado. Luego en la pestaña de Compartments se colocó el nombre del recinto, se añadió la geometría, es decir, el ancho, profundidad, altura y la posición relativa de los recintos a partir de las coordenadas X, Y, Z. Además, se señaló las características de los materiales de construcción de techo, paredes, suelo y las características de flujo. En esta pestaña Wall Vents se introdujo de cada compartimento los siguientes parámetros: a) Sill: Distancia de la apertura al suelo, b) Soffit: Distancia hacia la altura superior del marco de la puerta o ventana, c) Width: Ancho de la puerta o ventana, d) Vent offset: Distancia del eje x de la puerta o ventana a la esquina inferior izquierda del compartimento e) Face: Si la puerta o ventana está en la pared posterior, frontal, izquierda o derecha, f) Tiempo: Fracción de apertura de la ventana (Butafuoco et al.). Posteriormente se establecieron las características que tendrá la curva de fuego a simular para objetos como silla tapizada, armario, sofá, aparatos electrónicos y papel aquí se introdujo: a) Compartimento donde se producirá el incendio, b) Posición X, Y, Z en el recinto del fuego, c) Tiempo en el que inició el fuego, d) Curva HRR (Lacasta Palacio et al., 2019) la cual tiene dos connotaciones un antes para agua y un después para el ignífugo (CITA) e) Se insertó datos de moléculas de gases combustibles (índices medios molares) y calor de combustión en $\mathrm{KJ} / \mathrm{Kg}$. Finalmente se introdujo los sistemas de detección y supresión calculados introduciendo: a) Tipo de dispositivo: Sprinklers, b)Tipo de compartimento: Se seleccionó el compartimento precargado anteriormente por el usuario, c) Coordenadas: Ubicación de los sprinklers 23, d) Temperatura de activación: se utilizó una temperatura de $70^{\circ} \mathrm{C}$ tomada de la norma NFPA13 como se muestra en la Tabla 8 , e) RTI (Perez Piñas, 2016): Tiempo de respuesta característico del rociador (RTI) en (m s) ^0.5 según la Norma NFPA 13 se utilizó un valor de 80 (m s) ^ 0.5 , f) Densidad de pulverización incendios: Densidad de diseño en $\mathrm{m} / \mathrm{s}$ que será la cantidad de agua capaz de suministrar el rociador (Catalina \& Gonzalo, 2013) determinado a partir de la Tabla 9 tomada de la norma UNE 12845.

\section{Resultados}

\section{Simulación de sistema contra incendios, saturación de humo en el recinto}

En la tabla 1. Se observa la simulación con CFAST 7. del tiempo de saturación de humo en los recintos donde se activaron los sprinklers más rápido y más lento que en todos los emplazamientos.

Tabla 1. Tiempo de activación de los sprinklers en cada compartimento

\begin{tabular}{|c|c|c|c|}
\hline $\begin{array}{l}\text { Número de } \\
\text { sprinkler }\end{array}$ & $\begin{array}{c}\text { Tiempo } \\
\text { de } \\
\text { saturació } \\
\text { n de } \\
\text { humo sin } \\
\text { ignífugo } \\
\text { (seg) }\end{array}$ & $\begin{array}{c}\text { Tiempo } \\
\text { de } \\
\text { saturació } \\
\text { n de } \\
\text { humo } \\
\text { con } \\
\text { ignífugo } \\
\text { (seg) }\end{array}$ & $\begin{array}{l}\% \text { de } \\
\text { disminució } \\
\text { n } \\
\text { saturación } \\
(\%)\end{array}$ \\
\hline $\begin{array}{l}\text { 20Administraci } \\
\text { ón }\end{array}$ & & 1200 & 35 \\
\hline
\end{tabular}


Nota: Se procedió a la comparación de los dos escenarios de incendio considerando una disminución de la cantidad de energía liberada de los materiales HRR en un valor de $8 \%$ por el uso del ignífugo inorgánico y un tiempo de simulación de 9000 segundos. La activación del sprinkler 20 fue a los $18 \mathrm{seg}$ en la administración y $553 \mathrm{seg}$ en el área aséptica. La superficie máxima por rociador es de $12 \mathrm{~m}^{2}$, una densidad de diseño mínima de $5 \mathrm{~mm} / \mathrm{min}$.

En la figura 1 se observa la evolución del humo en los recintos en el tiempo máximo de saturación sin ignífugo y con ignífugo

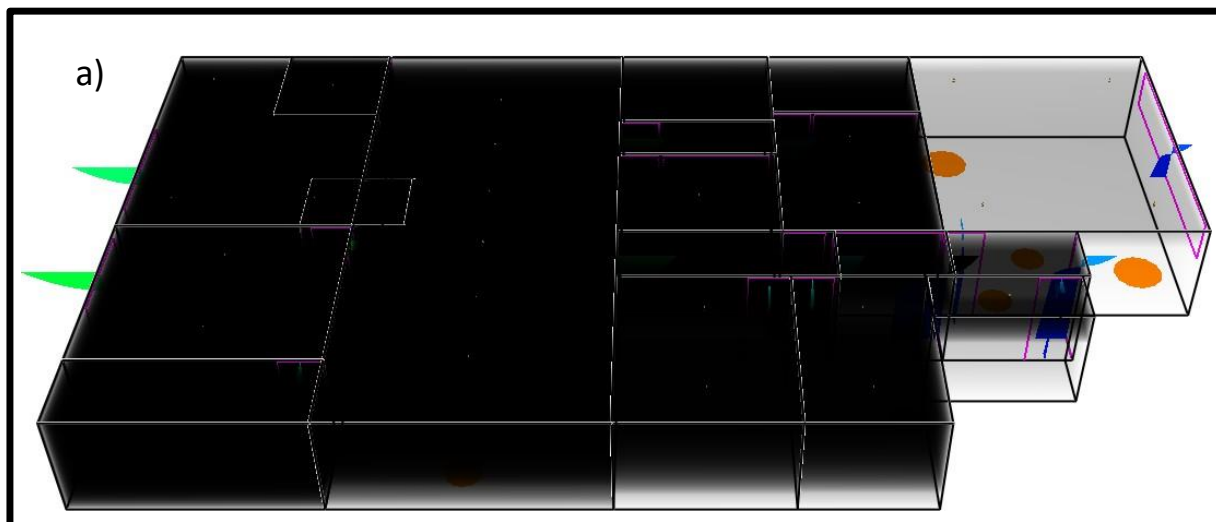

b)

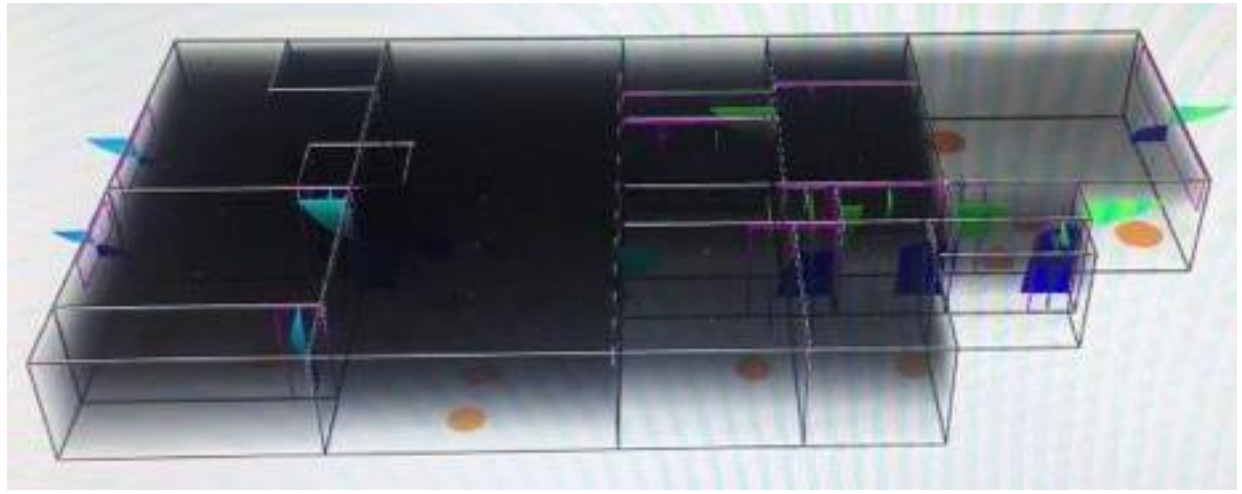

Figura 1. Simulación CFAST humo. a) Simulación de saturación de humo en recinto completo considerando extinción con agua b) Simulación de saturación de humo en recinto completo considerando extinción con agua

Simulación de sistema contra incendios, perfiles de temperaturas de las capas de productos

En la tabla 2. Se observa la simulación con CFAST 7. del tiempo transcurrido hasta que se enfría al activarse los medios de control de incendios. 
Tabla 2. Tiempo límite hasta que se produce el enfriamiento

\begin{tabular}{ccl}
\hline $\begin{array}{c}\text { Tiempo hasta } \\
\text { enfriamiento sin } \\
\text { ignífugo } \\
(\mathbf{s e g})\end{array}$ & $\begin{array}{c}\text { Tiempo hasta } \\
\text { enfriamiento con } \\
\text { ignífugo } \\
(\mathbf{s e g})\end{array}$ & $\begin{array}{l}\text { \% de disminución } \\
\text { tiempo de } \\
\text { enfriamiento } \\
(\boldsymbol{\%})\end{array}$ \\
\hline 350 & 180 & 48,57
\end{tabular}

Nota: Se procedió a la comparación de los dos escenarios de incendio considerando una disminución de HRR en un valor de $8 \%$ por el uso del ignífugo inorgánico y un tiempo de simulación de 9000 segundos. La capa caliente se observa de color cercano al rojo y la fría al azul.

En la figura 2 se observa la evolución de la temperatura en los recintos en función del tiempo hasta producirse 1 cambio al enfriamiento.

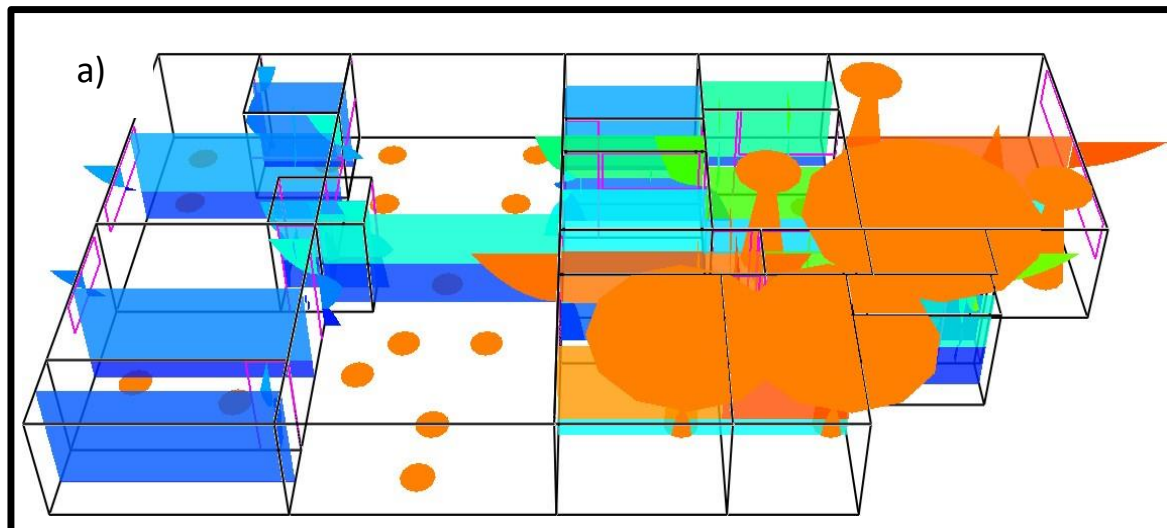

b)

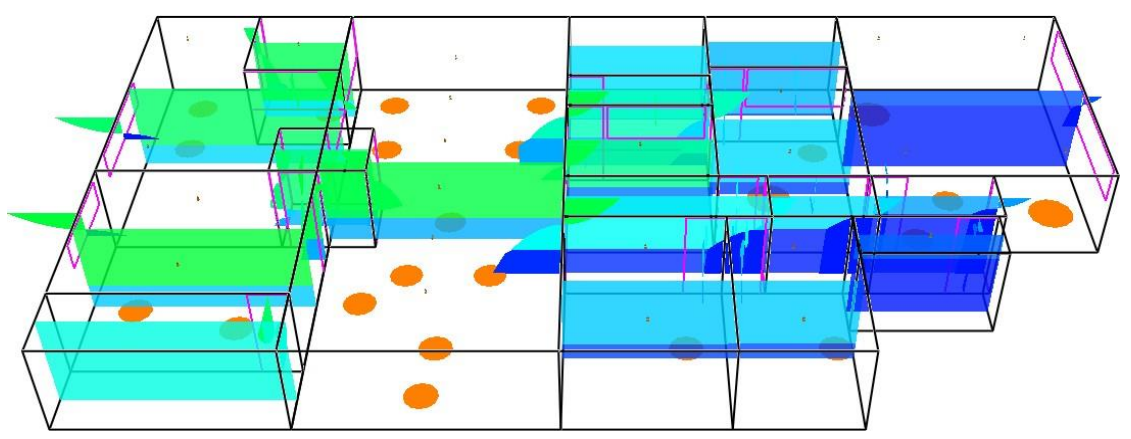

Figura 2. Simulación CFAST temperatura. a) Simulación de los perfiles de temperatura considerando agua a los 350 seg. de iniciado el enfriamiento en el primer caso. b) Simulación de los perfiles de temperatura a los $350 \mathrm{seg}$. considerando ignífugos de iniciado el enfriamiento en el primer caso.

\section{Conclusiones}

- Según valores obtenidos para un área de $264.04 \mathrm{~m} 2$ con un caudal total de abastecimiento de $4119.02 \mathrm{~L} / \mathrm{min}$, un nivel de riesgo de incendio extra-grupo 2 según NFPA 13, se calculó un volumen de abastecimiento de agua de 596645.17 L, el compartimiento más vulnerable y que activa más rápido los sistemas de 
splinkers (18 seg) en el Laboratorio químico LACONAL es el área de administración.

- Se simularon el tiempo de saturación del emplazamiento considerando el uso de agua y de dilución de ignífugos (Hidróxido de Aluminio e Hidróxido de Magnesio) como medio de extinción del sistema contraincendios determinado un $35 \%$ de mejora en el incremento del tiempo, así mismo el tiempo de enfriamiento fue un $48,57 \%$ menor que en condiciones normales de extinción, por los resultados se asume una mejora en la eficiencia del sistema contra incendios al utilizar ignífugos inorgánicos.

\section{Referencias}

Butafuoco, H., Rodrigues, J. P. C. y Coelho, A. L. Control de humos y temperatura en grandes atrios-El caso portugués.

Canosa, G. y Giudice, C. A. (2016). Modernos sistemas ignífugos para maderas de baja densidad.

Cohen, R. C. Z., Prakash, M., O'Grady, J. G., McInnes, K. L. y Hoeke, R. K. (2019). CFAST: Modelling and visualisation to improve flood adaptation planning processes in coastal cities. Paper presented at the Australasian Coasts and Ports 2019 Conference.

Grill, R. A. (2018). Standardizing NFPA 13. Consulting-Specifying Engineer, 55(9), 4046.

Huang, S., Song, Z. y Li, B. (2012) Reliability research of building fire based on numerical simulation and uniform design. Vol. 368-373. Advanced Materials Research (pp. 665-672).

Lacasta Palacio, A. M., Haurie Ibarra, L. y Giraldo Forero, M. d. P. (2019). Comportamiento de reacción al fuego del corcho. Paper presented at the Congreso LIGNOMAD 19: libro de ponencias: congreso sobre Construcción con Madera y otros Materiales Lignocelulósicos.

MANSILLA, C. H. V. (2014). DISEÑO DE UNA RED DE AGUA PARA ACCIONAR SPRINKLERS CONTRA INCENDIOS PARA EL EDIFICIO LUIS CHRISTEN ADAMS. Universidad Austral de Chile.

Mikhailovsky, E. (2020). Methods of mathematical modeling of fire extinguishing systems and their implementation in the ISIGR software package. Paper presented at the E3S Web of Conferences.

Perez Piñas, J. G. (2016). Cálculo y selección de sistema de rociadores contra incendio.

Portilla, D. A., Urrutia, M. L., Santos, P. E. y Abreu, J. A. C. (2006). Estudio de la capacidad predictiva de la dinámica del incendio mediante modelado y simulación computacional: CFAST, OZONE, FDS. Montajes e instalaciones: Revista técnica sobre la construcción e ingeniería de las instalaciones, 36(401), 79-83.

Pozo Álvarez, D. S. (2020). Eficacia de ignífugos inorgánicos (hidróxido de aluminio, hidróxido de magnesio) en la velocidad de combustión de calamagrostis intermedia de páramos de Ecuador. Universidad Técnica de Ambato. Facultad de Ciencia e Ingeniería en Alimentos ....

Purasinghe, R., De Rosas, J. C., Mejia, G., Thomas, M. y Chen, X. (2021) Fires and Building Safety. Vol. 94. Lecture Notes in Civil Engineering (pp. 555-560). 
Runefors, M., Boström, P. y Almgren, E. (2018). Comparison of sprinkler activation times under flat and corrugated metal deck ceiling. Paper presented at the Journal of Physics: Conference Series.

Saavedra Castro, J. L. y Rosales España, M. A. (2016). Evaluación del Sistema Contra Incendio Del Bloque A Del Hospital General Monte Sinaí De 400 Camas. Universidad de Guayaquil: Facultad de Ciencias Matemáticas y Físicas ....

Vadlamuri, R. P. y Lilley, D. G. (2009). CFAST: Application to a two-storey house with parameter effects on smoke detectors. Paper presented at the 47th AIAA Aerospace Sciences Meeting including the New Horizons Forum and Aerospace Exposition.

Vadlamuri, R. P. y Lilley, D. G. (2010). A two-storey house: Temperature and smoke detector activation with various fires using the CFAST fire computer code. Paper presented at the 48th AIAA Aerospace Sciences Meeting Including the New Horizons Forum and Aerospace Exposition.

Willis, J. J. F. y Llaja, A. M. V. (2020). PROPUESTA DE GUÍA PARA LA ELABORACIÓN DE UNA MEMORIA DE CÁLCULO DE SISTEMAS DE AGUA CONTRA INCENDIO. INGENIERÍA: Ciencia, Tecnología e Innovación, $7(2)$.

Zurita Castro, F. B. (2020). Diseño de un sistema contra incendios mediante la utilización de la herramienta CFAST en el Laboratorio de Control y Análisis de Alimentos LACONAL. Universidad Técnica de Ambato. Facultad de Ciencia e Ingeniería en Alimentos

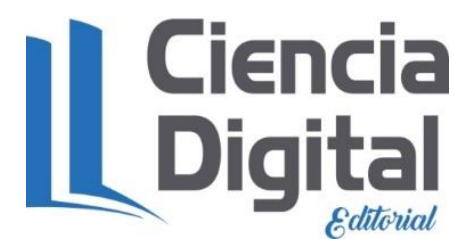




\section{PARA CITAR EL ARTÍCULO INDEXADO.}

Borja Mayorga, D. F., Córdova Suárez, M. A., Villacres Cevallos, E. P., \& Klever Saul, M. V. (2021). Simulación de la eficacia de ignífugos inorgánicos en la extinción de incendios con CFAST. Caso de un laboratorio químico. ConcienciaDigital, 4(1.2), 176186. https://doi.org/10.33262/concienciadigital.v4i1.2.1587

\section{Liencia}

El artículo que se publica es de exclusiva responsabilidad de los autores y no necesariamente reflejan el pensamiento de la Revista Conciencia Digital.

El artículo queda en propiedad de la revista y, por tanto, su publicación parcial y/o total en otro medio tiene que ser autorizado por el director de la Revista Conciencia Digital.

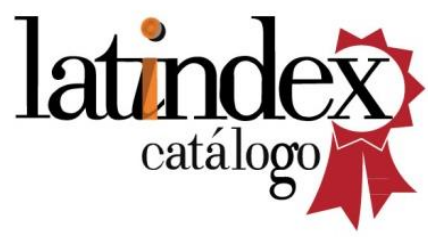

